\title{
Nuclear data measurement activities at CIAE
}

\author{
Xichao Ruanª, Liyang Jiang, Yi Yang, Shilong Liu, Yangbo Nie, Zhaohui Wang, Xiongjun Chen, Weixiang Yu, \\ and Zhigang Ge
}

Key Laboratory of Nuclear Data, China Institute of Atomic Energy, Beijing 102413, China

\begin{abstract}
China Institute of Atomic Energy (CIAE) is one of the most important bases for nuclear data measurement in China. In this paper, the nuclear data measurement activities at CIAE in recent years are presented. The secondary neutron emission DX and DDX measurement, the integral experiment for nuclear data benchmarking, the excitation function measurement, the neutron induced fission yields measurement and the gamma production yields measurement carried out in recent years are introduced. Furthermore, the progress of some new facilities and proposed plans (e.g. A Gamma ray Total Absorption Facility (GTAF) for $(n, \gamma)$ reaction cross section measurement, $\mathrm{A}^{3} \mathrm{He}$ detector array for $(\mathrm{n}, 2 \mathrm{n})$ reaction cross section measurement, the back streaming white neutron beam for nuclear data measurement at China Spallation Neutron Source (CSNS)) are also presented.
\end{abstract}

\section{Introduction}

The goal of nuclear data activities in China is supplying the nuclear data to feed the needs of the nuclear energy development, nuclear science study and nuclear technology applications. China Institute of Atomic Energy (CIAE) is one of the most important bases for nuclear data measurement in China. The facilities used for nuclear data measurement and study include the HI-13 tandem accelerator, $600 \mathrm{kV}$-Cockcroft-Walton accelerator and 5SDH-2 $2 \times 1.7 \mathrm{MV}$ tandem accelerator. In addition, the China Experimental Fast Reactor (CEFR, 65 MW) reached critical on 21 June 2010, and China Advanced Research Reactor (CARR, $60 \mathrm{MW}$, the maximum neutron flux: $8 \times 10^{14} \mathrm{n} / \mathrm{cm}^{2} \cdot \mathrm{s}$ ) reached critical on 13 , May 2010 at CIAE, were also used for nuclear data related research. The China Spallation Neutron Source(CSNS) [1,2] will also be put into operation in 2018. A white neutron source beam line $[3,4]$ based on CSNS will be available mainly for nuclear data measurement at that time. In this paper, the nuclear data measurement activities at CIAE in recent years are presented, and also the progress of some new facilities and proposed plans are given.

\section{Recent progress of nuclear data measurement at $\mathrm{CIAE}$}

\subsection{Excitation function measurements}

\section{1. ${ }^{69} \mathrm{Ga}(\mathrm{n}, 2 \mathrm{n}){ }^{68} \mathrm{Ga}$ cross section measurements.}

This measurement was performed with the $600 \mathrm{kV}$ Cockcroft-Walton accelerator in CIAE at the neutron energy of 14.1 and $14.9 \mathrm{MeV}$ with activation method. Based on the measurement, the existing experimental data of ${ }^{69} \mathrm{Ga}(\mathrm{n}, 2 \mathrm{n}){ }^{68} \mathrm{Ga}$ cross section were re-evaluated and a new evaluation was proposed based on CENDL-3.1.

\footnotetext{
a e-mail: xichao_ruan@126.com
}

In this work, the most important finding is that the $511 \mathrm{keV}$ gammas from the positron annihilation of ${ }^{68} \mathrm{Ga}$ are very difficult to measure accurately. Therefore the experimental data obtained by measuring $511 \mathrm{keV}$ gammas may be questionable. And the branching ratio of ${ }^{68} \mathrm{Ga}$ decay should be re-evaluated [5].

2. ${ }^{241} \mathrm{Am}(\mathrm{n}, \gamma)$ cross section measurement at thermal energy.

The experiment was carried out to measure the cross section of ${ }^{241} \mathrm{Am}(\mathrm{n}, \gamma)^{242 g} \mathrm{Am}$ reaction at the thermal neutron beam of the 49-2 swimming pool reactor in CIAE. The ${ }^{241} \mathrm{Am}$ sample was irradiated with a neutron beam with the flux of about $5 \times 10^{10} \mathrm{n} / \mathrm{cm}^{2} / \mathrm{s}$. After irradiation, the alpha particles from ${ }^{242} \mathrm{Am}$ were measured with a silicon detector. The preliminary result obtained in this work is $657 \pm 14 \mathrm{mb}$, and proved that this cross section is measurable with current experimental conditions.

\subsection{Fission yield measurement}

The fission yields of ${ }^{235} \mathrm{U}$ at $3 \mathrm{MeV}, 14 \mathrm{MeV}$ and ${ }^{252} \mathrm{Cf}$ spontaneous fission neutrons, ${ }^{232} \mathrm{Th}$ at $14 \mathrm{MeV}$ neutrons were measured. Fission product activities were measured by a HPGe $\gamma$-ray spectrometer. Fission yields were determined by method of $\mathrm{R}$-values relative to ${ }^{140} \mathrm{Ba}$. Combined with previous measurements, part of the energy dependent fission yield for $\mathrm{n}+{ }^{235} \mathrm{U}$ fission reaction was shown in Fig. 3. One can see that the fission yields for some energy points can deviate from a linear function more than $10 \%$. They agree with the systematic calculation result.

In addition, the technique for fission product mass yield distribution measurement is under development at CIAE. The E-v method of measuring the kinetic energy $(E)$ and velocity $(\mathrm{V})$ of outgoing fission products has been utilized, with the goal of measuring the mass resolution better than 1 atomic mass units(amu),could identify every mass for light fission products of unsymmetrical fission. 


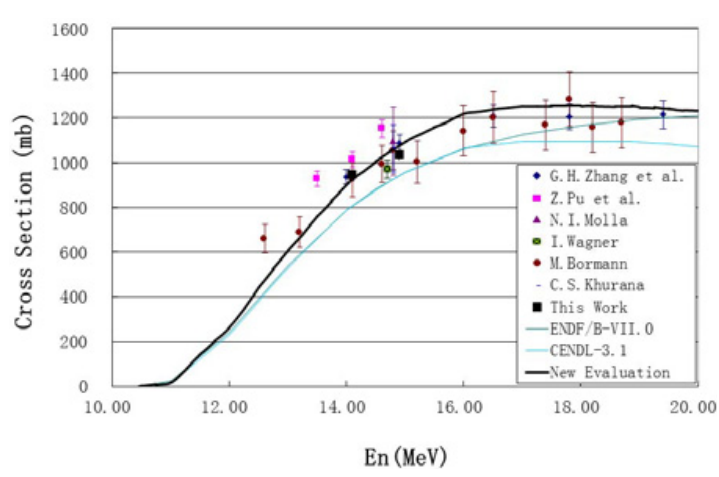

Figure 1. Measured result of ${ }^{69} \mathrm{Ga}(\mathrm{n}, 2 \mathrm{n}){ }^{68} \mathrm{Ga}$ cross section compared with other measurements and evaluations.

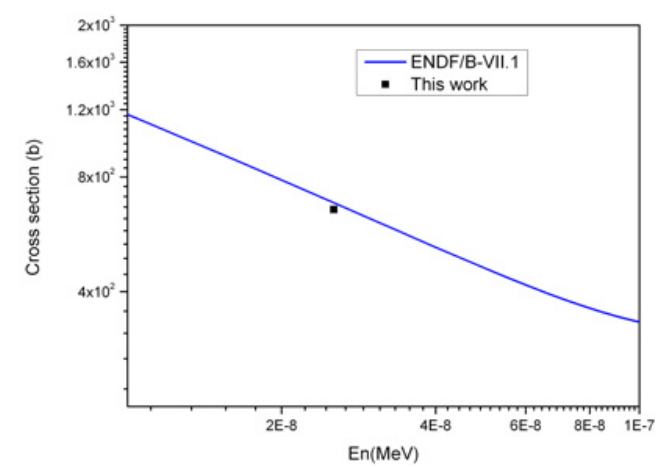

Figure 2. The preliminary result of ${ }^{241} \mathrm{Am}(\mathrm{n}, \gamma)^{242 g} \mathrm{Am}$ cross section at thermal energy.

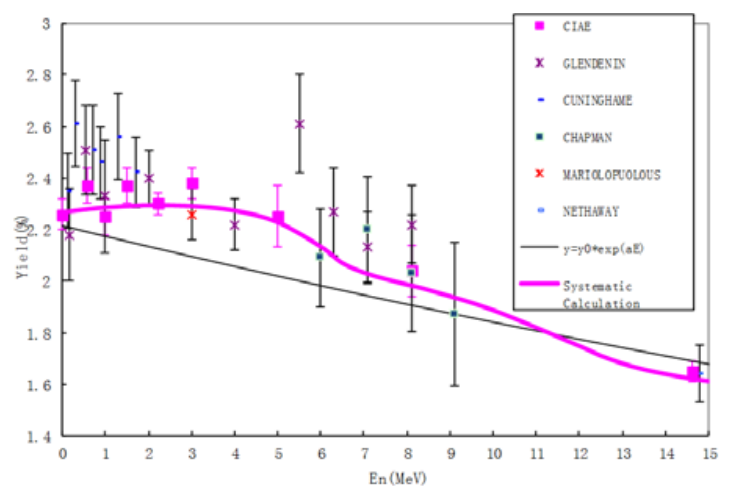

Figure 3. Energy dependent fission yields of ${ }^{147} \mathrm{Nd}$ from ${ }^{235} \mathrm{U}$ fissions.

\subsection{The secondary neutron emission double-differential cross section measurement}

The measurement of the double-differential cross sections (DDXs) of ${ }^{9} \mathrm{Be}$ at 22 and $25 \mathrm{MeV}$ neutron energies and the measurement of DDXs of D at $8 \mathrm{MeV}$ have been finished. These measurements are performed with the multi-detector fast neutron TOF spectrometer at the HI-13 Tandem Accelerator in CIAE. The diagram of the spectrometer is shown in Fig. 4. The $22 \mathrm{MeV}$ neutrons were produced by the $\mathrm{T}(\mathrm{d}, \mathrm{n})^{4} \mathrm{He}$ reaction with a tritium gas target, while the $8 \mathrm{MeV}$ neutrons were produced by $\mathrm{D}(\mathrm{d}, \mathrm{n})^{3} \mathrm{He}$ reaction with a deuterium gas target. The scattering samples were placed at $0^{\circ}$ with respect to the deuteron beam about $20 \mathrm{~cm}$ away from the centre of the gas target. A polyethylene sample was used to obtain the n-p scattering yield, and this yield was used for cross section normalization. More

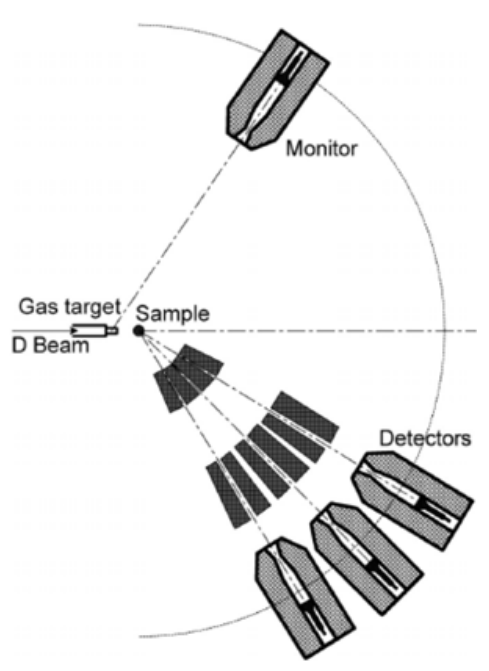

Figure 4. Schematic view of the multi-detector fast neutron TOF spectrometer at CIAE.

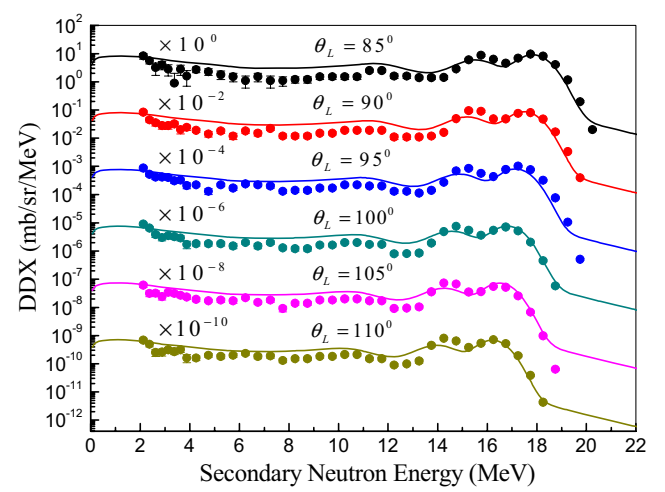

Figure 5. Part of the measured DDXs for Be at $22 \mathrm{MeV}$.

details about the experiments and the dada analysis can be found in Refs. [6-8].

Figure 5 shows part of the measured DDXs result of $\mathrm{Be}$ at $22 \mathrm{MeV}$, comparing with theoretical calculation with the LUNF code.

\subsection{The gamma production yields measurement}

A HPGe detector array consists of 4 Clover detectors and 4 planar HPGe detectors was developed for this purpose. The detection system was used to measure the prompt $\gamma$ rays from $(\mathrm{n}, \mathrm{xn} \gamma)$ and $\left(\mathrm{n}, \mathrm{n}^{\prime} \gamma\right)$ reaction. Based on the $600 \mathrm{kV}$ Cockcroft-Walton accelerator in CIAE, the measurements for ${ }^{\text {nat }} \mathrm{Fe}\left(\mathrm{n}, \mathrm{n}^{\prime} \gamma\right)$ and ${ }^{235,238} \mathrm{U}(\mathrm{n}, 2 \mathrm{n} \gamma)$ have been carried out. Pulsed and direct current beam have been used to study the time dependent backgrounds. With the measurement of nat Fe target, the detection system was validated. In the experiment of ${ }^{238} \mathrm{U}$ target, A few $\gamma$ rays from ${ }^{238} \mathrm{U}(\mathrm{n}, \mathrm{xn})$ reactions have been observed, and the cross sections obtained are consistent with others' measurements. The data analysis of ${ }^{235} \mathrm{U}(\mathrm{n}, \mathrm{xn} \gamma)$ is ongoing.

\subsection{Nuclear data benchmark experiments}

The aim of the experiments is to make nuclear data benchmark for various important materials for the R\&D of new generation nuclear energy systems such as CIADS(Chinese Initiative Accelerator Driven System) and TMSR in China. For this purpose, the neutron leakage 


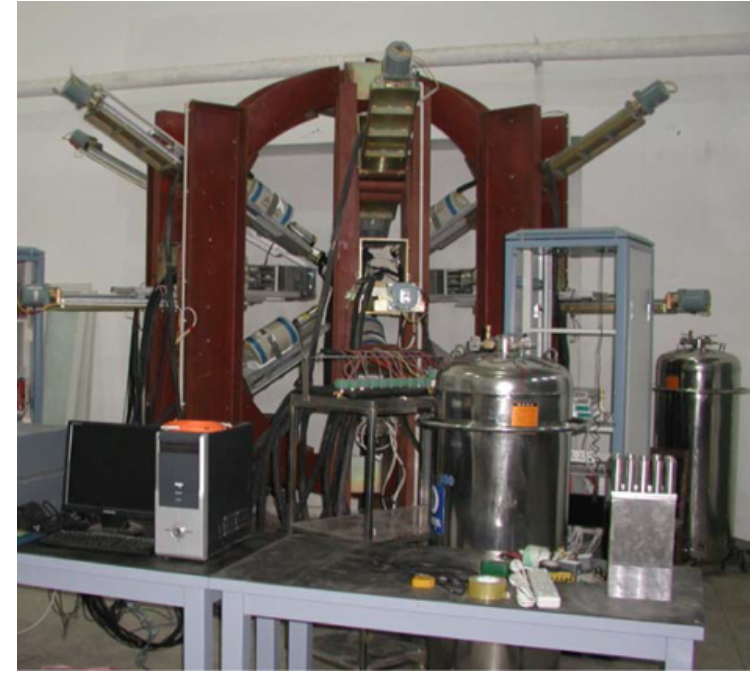

Figure 6. The HPGe detector array at CIAE.

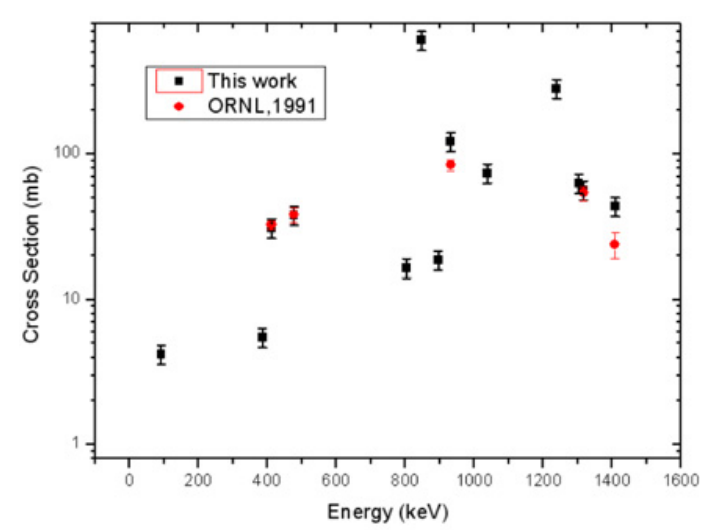

Figure 7. The measured results for ${ }^{56} \mathrm{Fe}\left(\mathrm{n}, \mathrm{n}^{\prime} \gamma\right)$.

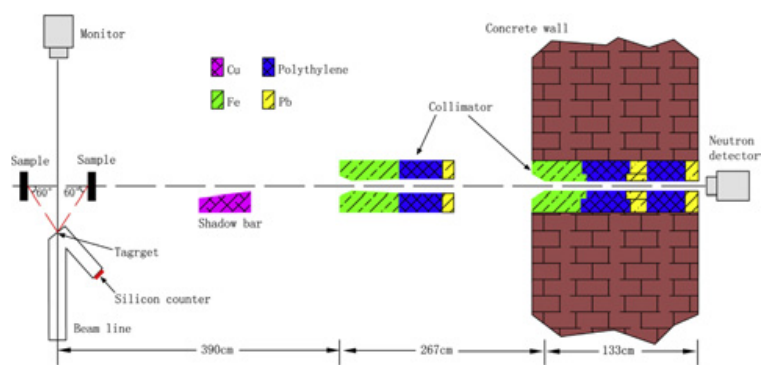

Figure 8. The experimental arrangement for the integral experiments.

spectra in the energy range from 0.8 to $15 \mathrm{MeV}$ from slab samples were measured by time-of-flight technique with a BC-501A scintillation detector. The measurements were performed at China Institute of Atomic Energy (CIAE) using a d-T neutron source. Figure 8 shows the experimental arrangement. The thicknesses of the slabs were 0.5 to 2.5 mean free paths for $14.8 \mathrm{MeV}$ neutrons, and the measured angles were chosen to be $60^{\circ}$ and $120^{\circ}$. The measured spectra were compared with those calculated by the continuous energy Monte-Carlo transport code MCNP, using the data in the CENDL-3.1, ENDF/B-VII.1 and JENDL-4.0 nuclear data files. Table 1 list the measured samples in recent years. More details can be found in Refs. [9-12].
Table 1. The measured samples.

\begin{tabular}{|l|l|c|l|}
\hline Sample & Sample size/cm & Sample thickness $/ \mathrm{cm}$ & Angle $^{\circ}$ \\
\hline${ }^{238} \mathrm{U}$ & $10 \times 10$ & 5 & 45,135 \\
\hline $\mathrm{Be}$ & $10 \times 10$ & 5,11 & 60,120 \\
\hline${ }^{\mathrm{n}} \mathrm{Ga}$ & $\Phi 13$ & $3.2,6.4$ & 60,120 \\
\hline${ }^{\mathrm{n}} \mathrm{W}$ & $10 \times 10$ & $3.5,7$ & 60,120 \\
\hline Granular $\mathrm{W}$ & $10 \times 10$ & 5 & 60 \\
\hline $\mathrm{C}$ & $\Phi 13$ & 20 & 60,120 \\
\hline $\mathrm{SiC}$ & $\Phi 13$ & 20 & 60,120 \\
\hline $\mathrm{Pb}$ & $\Phi 13$ & 5 & 60 \\
\hline $\mathrm{Pb}-\mathrm{Bi}$ & $\Phi 13$ & 5 & 60 \\
\hline $\mathrm{ThO}_{2}$ & $\Phi 13$ & $5.4,10.8$ & 60,120 \\
\hline $\mathrm{H}_{2} \mathrm{O}$ & $\Phi 13$ & 5.2 & 60 \\
\hline $\mathrm{PE}$ & $\Phi 1310 \times 10$ & 65 & 6045 \\
\hline
\end{tabular}

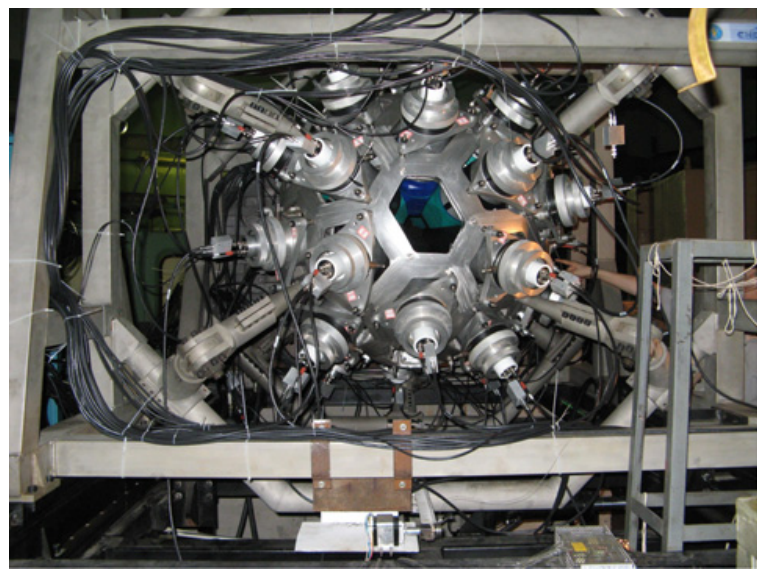

Figure 9. The GTAF facility at CIAE.

\section{New facilities for nuclear data measurement}

\subsection{The gamma ray total absorption facility (GTAF)}

To feed the needs of neutron capture cross section measurement, the Gamma ray Total Absorption Facility (GTAF) has been constructed [13]. The detector consists of $40 \mathrm{BaF}_{2}$ crystals. The neutron capture event detection efficiency is expected to be more than $95 \%$ with this facility. The construction of this facility is now finalized and the test physics run is undergoing.

\section{2. ${ }^{3} \mathrm{He}$ detector array for $(n, 2 n)$ reaction cross section measurement}

Traditionally, the activation method was used to measure the $(n, 2 n)$ cross sections. The advantage of this method is that the experimental setup is simple and high precise data can be obtained. But the disadvantage is that the half-life of the compound nuclei has to be suitable for offline measurement, which blocks the measurement of a lot of interesting nucleus. To overcome this disadvantage, a detector array consists of $100{ }^{3} \mathrm{He}$ proportional counters has been developed. Figure 10 shows the detector system. Spherical design was adopted to make the detection efficiency more independent of neutron energy. The neutron detection efficiency is about $30 \%$ for neutron energy below $5 \mathrm{MeV}$. 


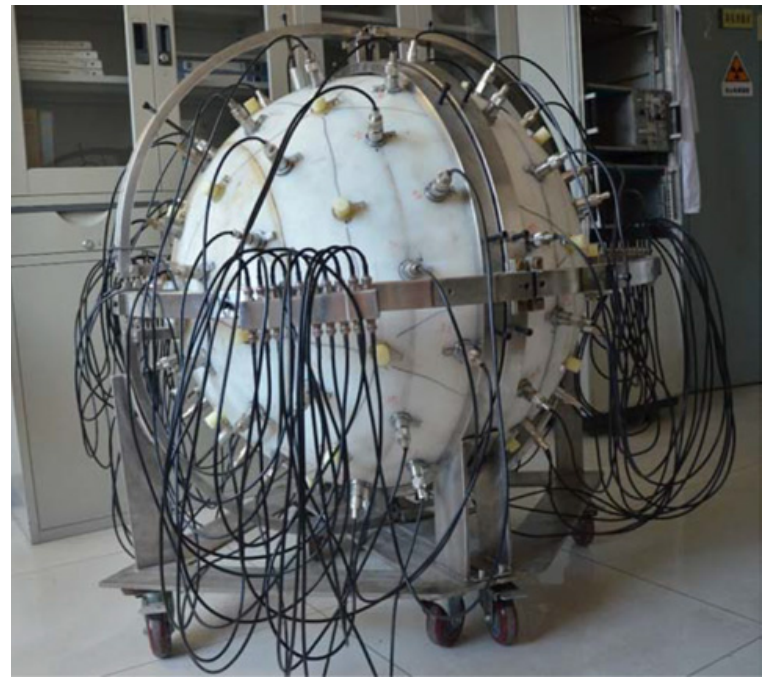

Figure 10. The ${ }^{3} \mathrm{He}$ detector array.

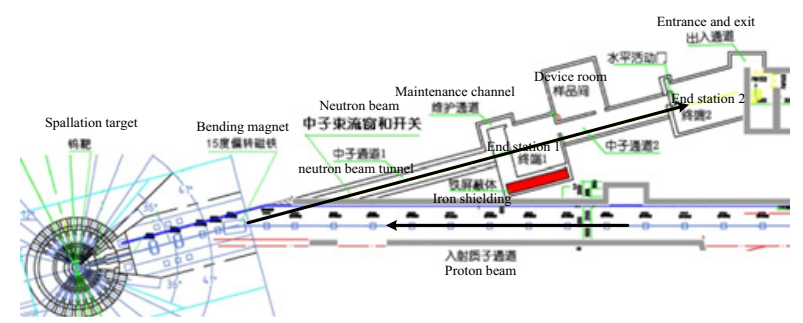

Figure 11. Layout of the CSNS back-streaming neutron beam tunnel and the experimental halls.

\subsection{The back-streaming neutron beam line at CSNS}

The China Spallation Neutron Source (CSNS) is a large scientific facility dedicated mainly to multidisciplinary research on material characterization using neutron scattering techniques. This project was officially approved by the Chinese central government in 2008, and will be in operation in 2018 for the $1^{\text {st }}$ stage. In the first phase (CSNS-I), the machine will deliver $1.6 \mathrm{GeV}$ protons on a tungsten target (TS1) with $100 \mathrm{~kW}$ beam power and a pulse repetition rate of $25 \mathrm{~Hz}$. A back-streaming neutron beam line based on this machine was proposed and constructed mainly for nuclear data measurements.

A detailed study of the characteristics for the backstreaming neutrons at CSNS is described in Ref. [4]. Based on this study, a nuclear data measurement program using the CSNS back-streaming neutron beam was proposed. The layout of the neutron beam tunnel and the experimental halls are shown in Fig. 11. Two end stations were constructed, with $\sim 50$ meters flight path for end station 1 and $\sim 80$ meters for end station 2 , respectively. The end station 1 will be used for higher neutron flux required experiment while the end station 2 for better time resolution required experiment. In the first stage, the neutron total cross section measurement, capture cross section measurement with C6D6 detectors and fission cross section measurement with a normal ionization chamber are planed with this back-streaming neutron beam.

\section{Conclusion}

Substantial progress on nuclear data measurement has been made in China in recent years. More and more needs for nuclear data measurement have been required with the progress of the CIADS, TMSR and ITER projects in China. Some new facilities such as CSNS are under construction. These facilities will greatly improve the capability of the nuclear data measurement in China in the near future.

This work is supported by the Key Research Program from the Ministry of Science and Technology of China (2016YFA0401601) and the Advanced Research Project for nuclear data under grant No. 4160303.

\section{References}

[1] S.X. Fang, et al., J. Kor. Phys. Soc. 48, 697 (2006)

[2] H.S. Chen and X.L. Wang, Nature Materials 15, 689 (2016)

[3] J.Y. Tang, et al., Chinese Physics C 34(1), 121 (2010)

[4] H.T. Jing, et al., Nuclear Instruments and Methods in Physics Research A621, 91 (2010)

[5] X.L. Huang, et al. Chinese Physics C 38(4), 044001-1 (2014)

[6] J. Sa, et al., Atomic Energy Sci. Technol. 26(6), 1 (1992) (in Chinese)

[7] D. Schmidt, et al. Nuclear Instruments and Methods in Physics Research A545, 658 (2005)

[8] X.C. Ruan, et al. Chinese Physics C 31(5), 442 (2007)

[9] Y. Nie, et al. Ann. Nucl. Energy 37, 1456 (2010)

[10] R. Han, et al. Nucl. Phys. A 936, 17 (2015)

[11] S. Zhang, et al. Fus. Eng. Des. 92, 41 (2015)

[12] Y. Nie, et al. Fus. Eng. Des. 105, 8 (2016)

[13] Q.P. Zhong, et al. Chinese Physics C 32(suppl. II), 102 (2008) 